\title{
SÍNTESIS DE CATALIZADORES BASADOS EN ÓXIDOS MIXTOS DE Ni-Ti PARA LA PRODUCCIÓN DE ETILENO A PARTIR DE LA DESHIDROGENACIÓN CATALÍTICA DEL ETANO
}

\author{
Daisy Unsihuay ${ }^{\mathrm{a}}$ Gino Picasso ${ }^{\mathrm{a}^{*}}$, Rosario Sun Kou ${ }^{\mathrm{b}}$
}

\begin{abstract}
RESUMEN
Se prepararon catalizadores a base de óxidos de níquel dopados con titanio $\mathrm{Ni}_{1-\alpha} \mathrm{Ti}_{\alpha} \mathrm{O}$ con $\alpha$ de 0,01 a 0,05 , por un método de evaporación controlada a $550^{\circ} \mathrm{C}$ para la deshidrogenación oxidativa (ODH) de etano a etileno. La introducción de pequeñas cantidades de Ti en la celda del $\mathrm{NiO}$ tuvo efectos notables sobre las propiedades de óxido simple de níquel, aumentando la superficie BET. Además, se observó una disminución del tamaño del cristal tras el análisis $\mathrm{XRD}$, una generación de nuevos sitios ácidos, evaluados por TPD-NH${ }_{3}$, una redistribución de especies reducibles a temperaturas más bajas y más altas estudiadas a partir del perfil TPR- $\mathrm{H}_{2}$ y una disminución de los oxígenos no estequiométricos con la composición creciente de titanio detectada por TPD- $\mathrm{O}_{2}$. Los ensayos catalíticos se llevaron a cabo en condiciones de estado estacionario a una constante $\mathrm{W} / \mathrm{F}$ de $0,48 \mathrm{~g} . \mathrm{s} / \mathrm{mL}$ con el fin de evaluar la conversión y se modificó la constante W/F de 0,24 a 0,48 g.s / $\mathrm{mL}$, para cuantificar la selectividad al etileno. En todos los catalizadores $\mathrm{Ni}_{1-\alpha} \mathrm{Ti}_{\alpha} \mathrm{O}$, las pruebas catalíticas revelaron un efecto positivo de dopaje de Ti en el rendimiento de las muestras, mejorando los niveles de conversión y de selectividad de etano al etileno, en comparación con el $\mathrm{NiO}$ puro. La muestra $\mathrm{Ni}_{0.95} \mathrm{Ti}_{0.05} \mathrm{O}$ logró la mejor selectividad para el etileno $(77 \%)$ en una conversión moderada de etano del $35 \%$, probablemente debido al aumento de las especies nucleofílicas de oxígeno superficial.
\end{abstract}

Palabras clave: ODH de etano, conversión de etano, selectividad de etileno.

\section{SYNTHESIS OF CATALYSTS BASED ON Ni-Ti MIXED OXIDES FOR THE PRODUCTION OF ETHYLENE FROM CATALYTIC DEHYDROGENATION OF ETHANE}

\begin{abstract}
Catalysts based on titanium doped nickel oxides $\mathrm{Ni}_{1-\alpha} \mathrm{Ti}_{\alpha} \mathrm{O}$ with $\alpha$ from 0.01 to 0.05 , were prepared by a controlled evaporation method at $550^{\circ} \mathrm{C}$ for selective oxidative dehydrogenation $(\mathrm{ODH})$ of ethane to ethylene. The introduction of small amounts of Ti into the NiO lattice

\footnotetext{
${ }^{a}$ Laboratorio de Investigación de Fisicoquímica, Facultad de Ciencias, Universidad Nacional de Ingeniería, Av. Túpac Amaru 210, Rímac, Lima, Perú, *gpicasso@uni.edu.pe

${ }^{\mathrm{b}}$ Departamento de Ciencias-Sección Química, Pontificia Universidad Católica del Perú, Avenida Universitaria 1801 - Lima 32.
} 
had noticeable effects on the nickel simple oxide properties, increasing the BET surface. In addition, it was observed a sustained depletion of crystallite size following XRD analysis, a generation of new acid sites, evaluated by NH3-TPD, a redistribution of reducible species to lower and higher temperatures studied from $\mathrm{H}_{2}$-TPR profile and a depression of nonstoichiometric oxygen with the increasing composition of titanium detected by $\mathrm{O}_{2}$-TPD. The catalytic tests were carried out under steady state conditions at a constant W/F of 0.48 g.s/ $\mathrm{mL}$ in order to evaluate the conversion and modifying $\mathrm{W} / \mathrm{F}$ from 0.24 to $0.48 \mathrm{~g} . \mathrm{s} / \mathrm{mL}$, to quantify the selectivity to ethylene. In all the Ni1- $\alpha \mathrm{Ti} \alpha \mathrm{O}$ catalysts, the catalytic tests revealed a positive doping effect of $\mathrm{Ti}$ in the performance of the samples, improving the ethane conversion and selectivity levels to ethylene, in comparison to the pure NiO. The sample $\mathrm{Ni}_{0.95} \mathrm{Ti}_{0.05} \mathrm{O}$ achieved the best selectivity to ethylene $(77 \%)$ at a moderated ethane conversion of $35 \%$, probably due to the increasing of the nucleophilic species of surface oxygen.

Key words: ODH, ethane, Ti-doped $\mathrm{NiO}$, ethylene selectivity.

\section{INTRODUCCIÓN}

El etileno es la materia prima orgánica de mayor consumo en la industria química y se espera que su demanda siga en aumento en los próximos años. En este contexto, es necesario el desarrollo de nuevas tecnologías para la producción de etileno con el fin de satisfacer las demandas esperadas de los próximos años ${ }^{1}$. La deshidrogenación oxidativa (ODH) de etano es una ruta alternativa atractiva ya que opera a bajas temperaturas $\left(-400^{\circ} \mathrm{C}\right)$, es económica y amigable con el medio ambiente en comparación con el craqueo al vapor, un proceso altamente endotérmico que opera por encima de $800^{\circ} \mathrm{C}$ y hoy en día el principal método de obtención de etileno ${ }^{2}$. Sin embargo, el logro de altos niveles de conversión de etano y una alta selectividad a etileno son particularmente difíciles debido a que las olefinas tienden a oxidarse a $\mathrm{CO}_{2}$ y $\mathrm{CO}$. Este último, es la principal causa de envenenamiento del catalizador y disminuye drásticamente su rendimiento ${ }^{3}$. Por lo tanto, el desarrollo de un catalizador activo y selectivo para la conversión de etano a etileno a bajas temperaturas es clave para que la ODH de etano sea competitiva con el craqueo a vapor. A lo largo de los últimos años, un gran número de catalizadores basados en metales de transición se han preparado de los cuales los catalizadores basados en níquel han reportado alcanzar buenos niveles de conversión de etano, sin embargo, la selectividad es todavía una tarea difícil de mejorar. La incorporación de promotores en la red de $\mathrm{NiO}$ tales como $\mathrm{Ta}, \mathrm{Nb}, \mathrm{Ce}, \mathrm{Co}, \mathrm{W}$ y Al aumentó el rendimiento de los catalizadores basados en $\mathrm{NiO}^{4-9}$. Heracleous y col. ${ }^{6}$, reportaron que la adición de promotores con valencia mayor que $\mathrm{Ni}^{2+}$ tuvo un efecto positivo en el logro de buenas selectividades ya que eran capaces de disminuir los defectos catiónicos, y por lo tanto, las especies de oxígeno no selectivos que conllevan a la sobreoxidación de la olefina. En el conjunto de catalizadores ensayados, el único catalizador a base de Ni-Ti exhibió buenos niveles de selectividad (85\%) pero, por el contrario, la conversión de etano (10\%) era muy pobre. En el presente trabajo, en base a los prometedores resultados reportados en la literatura sobre catalizadores de NiTi-O, hemos explorado el papel de catalizadores de dopaje $\mathrm{NiO}$ con diferentes cantidades de Ti con el fin de mejorar los niveles de actividad y selectividad. Un método de evaporación 
simple pero controlada se realizó con el fin de favorecer la integración de fases para formar una solución mixta.

\section{PARTE EXPERIMENTAL}

\section{Síntesis de los catalizadores}

Solución de isopropóxido (trietanolaminato) de titanio (IV) $\left(\mathrm{C}_{9} \mathrm{H}_{19} \mathrm{NO}_{4} \mathrm{Ti}\right)$ (80 wt.\% en isopropanol) se adquirió de Aldrich y nitrato de níquel hexahidratado $\left(\mathrm{Ni}\left(\mathrm{NO}_{3}\right)_{2} \cdot 6 \mathrm{H}_{2} \mathrm{O}\right)$ níquel (II) se adquirió de Merck ( $99 \%$ de pureza), ambos reactivos fueron usados como precursores de los óxidos mixtos sin purificación adicional. Etanol (Merck, pureza 99,9\%) fue empleado como un disolvente.

Una serie de catalizadores con diferente composición atómica $\mathrm{Ni}_{1-\alpha} \mathrm{Ti}_{\alpha} \mathrm{O}$ con $(\alpha=0,0,01$, $0,03,0,04,0,05$ y 1 ) se prepararon mediante el método de evaporación. Debido a que $\mathrm{TiO}_{2}$ puede formarse directamente a partir de la hidrólisis del alcóxido de titanio en un medio acuoso, el etanol se empleó como solvente para las soluciones precursoras para moderar la alta reactividad y $\mathrm{C}_{9} \mathrm{H}_{19} \mathrm{NO}_{4} \mathrm{Ti}$ se utilizó como precursor de Ti a fin de proporcionar más estabilidad al sistema y evitar la segregación de fases durante la síntesis. Las soluciones etanólicas de $\mathrm{C}_{9} \mathrm{H}_{19} \mathrm{NO}_{4} \mathrm{Ti}$ y $\mathrm{Ni}\left(\mathrm{NO}_{3}\right)_{2} \cdot 6 \mathrm{H}_{2} \mathrm{O}$ se prepararon por separado y se utilizaron inmediatamente para la síntesis. La solución de Ti se añadió gota a gota a la solución de Ni con agitación vigorosa a temperatura ambiente y se mantuvo en las mismas condiciones durante 30 minutos. Después, la evaporación del etanol se llevó a cabo a $80{ }^{\circ} \mathrm{C}$ hasta que se obtuvo un gel verde. Luego, el gel se dejó secar a $120^{\circ} \mathrm{C}$ durante 12 horas, seguido de calcinación con aire a $550{ }^{\circ} \mathrm{C}$ a una velocidad de calentamiento de $2{ }^{\circ} \mathrm{C} / \mathrm{min}$ durante 4 horas.

\section{Caracterización de los catalizadores}

Las propiedades texturales de los sólidos fueron estudiadas utilizando el equipo Micromeritics GEMINI VII a la temperatura de nitrógeno líquido. Las muestras se trataron previamente en el vacío por la desgasificación a $250^{\circ} \mathrm{C}$ durante $2 \mathrm{~h}$. El área superficial se calculó de acuerdo con el método BET. La distribución de tamaño de poro y tamaño del poro medio se estimaron por el método de análisis BJH aplicado a la rama de desorción de la isoterma. El análisis elemental por espectroscopia de rayos $\mathrm{X}$ de energía dispersiva (EDX) se llevó a cabo para determinar las composiciones de los catalizadores.

La estructura cristalina de las muestras fue evaluada por difracción de rayos X (XRD). El análisis se realizó con un difractómetro Siemens D5000 Krystalloflex, que opera con los siguientes parámetros: la radiación $\mathrm{Cu} \mathrm{K} \alpha(\lambda=1,5418 \AA), 15 \mathrm{~mA}, 30 \mathrm{kV}$, filtro de $\mathrm{Ni}$, ángulo $2 \theta$ en un rango de 10 a $90^{\circ}$ con una velocidad de $0,04^{\circ}$ s. El tamaño de cristal se estimó usando la ecuación de Scherrer de la señal más intensa difracción de rayos X.

La capacidad reductora de las muestras fue analizada por reducción a temperatura programada (TPR) en el equipo Micromeritics ChemiSorb 2750. Los experimentos se realizaron en un reactor con $50 \mathrm{mg}$ de catalizadores bajo atmósfera de hidrógeno diluido ( $5 \%$ de $\mathrm{H}_{2}$ en $\left.\mathrm{He}\right)$ de 30 a $850^{\circ} \mathrm{C}$ a una velocidad de calentamiento de $10^{\circ} \mathrm{C} / \mathrm{min}$. 
La acidez de las muestras se midió a través de una desorción a temperatura programada de $\mathrm{NH}_{3}$. Las muestras se cargaron en un tubo de cuarzo en forma de U (50 mg). El tratamiento previo de la muestra se llevó a cabo de la siguiente manera: una purga con helio se hizo durante una hora a $100^{\circ} \mathrm{C}$. Después, el gas se cambió a amoníaco para dar paso a la adsorción durante 30 minutos a la misma temperatura y finalmente se hizo otra purga con helio durante una hora para eliminar las moléculas de amoníaco que no fueron adsorbidas por la muestra. Después se procedió a calentar el horno con una rampa de $15{ }^{\circ} \mathrm{C} / \mathrm{min}$ desde $100{ }^{\circ} \mathrm{C}$ a 600 ${ }^{\circ} \mathrm{C}$ bajo un flujo de helio y se mantuvo a la temperatura final durante media hora hasta que la señal regresó a la línea de base. La cantidad desorbida de $\mathrm{NH}_{3}$ se monitorizó mediante un detector de conductividad térmica (TCD), utilizando el software V1.03 TPX ChemiSoft.

\section{Ensayos catalíticos}

Los estudios de reactividad de los catalizadores se realizaron en un reactor de lecho fijo de cuarzo. Una pequeña cantidad de lana de cuarzo se colocó en la frita de vidrio para obstaculizar el flujo del catalizador a través del tubo de cuarzo. Para cada catalizador, 200 mg de muestra se mezclaron con $400 \mathrm{mg}$ de cuarzo con el fin de favorecer una distribución homogénea de la temperatura. Un separador de agua se instaló al lado del horno para capturar el agua que puede ser producida como un subproducto de la ODH etano. La composición del flujo de alimentación de la reacción fue de $0,25 \% \mathrm{C}_{2} \mathrm{H}_{6}, 0,25 \%$ de $\mathrm{O}_{2} \mathrm{y} \mathrm{N}_{2}$ como gas de equilibrio. Los experimentos conversión de etano se realizaron en el intervalo de temperatura de $300-450^{\circ} \mathrm{C}$, en una proporción constante $\mathrm{W} / \mathrm{F}$ de $0,48 \mathrm{gs} / \mathrm{mL}$. La selectividad de etileno frente a la conversión de etano se midió a una temperatura fija de $450{ }^{\circ} \mathrm{C} \mathrm{y} \mathrm{W/F}$ varió de 0,24 a 2,4 gs/mL. Para todas las muestras, la conversión de etano, y los niveles de selectividad de etileno se calcularon basándose en el balance de carbono con un error experimental inferior al $5 \%$ para todas las medidas.

\section{RESULTADOS Y DISCUSIÓN}

\section{1.- Caracterización por sorción de $N_{2}$ y XRD}

La relación metálica de los Ni-Ti-O se determinó por EDX. Las composiciones estequiométricas teóricos para los catalizadores fueron aproximados con los resultados experimentales (tabla 1). Las isotermas de adsorción-desorción de $\mathrm{N}_{2}$ están mostradas en la figura 1. Los catalizadores de $\mathrm{NiO}$ y Ni-Ti-O exhibieron la típica isoterma de tipo IV correspondiente a sólidos micro-mesoporosos donde se produce adsorción en multicapas. Se puede apreciar que las isotermas poseen histéresis de tipo $\mathrm{H}_{3}$ originada por condensación capilar que solo se da cuando se presentan mesoporos (poros de 2 a $50 \mathrm{~nm}$ ). Este tipo de histéresis es característica de materiales compuestos por partículas de poros flexibles con morfología de tipo rendija. Por otro lado el $\mathrm{TiO}_{2}$ mostró una isoterma de tipo IV con histéresis del tipo H2 causado cuando la distribución de tamaño y la forma de los poros no están bien definidos y también indicativo de constricciones de cuello de botella. Las áreas BET (tabla 1) registradas para los catalizadores de $\mathrm{NiO}$ fueron relativamente pequeñas en el rango de 8-46 $\mathrm{m}^{2} \mathrm{~g}^{-1}$ pero acorde a lo esperado por el método de evaporación usado para la preparación de los catalizadores ya que no tiende a producir áreas superficiales grandes en comparación 
a otros métodos como sol-gel, precipitación, etc. Se observó un aumento notorio del área superficial del $\mathrm{NiO}$ a mayor contenido de Ti. Este aumento posiblemente ocasionado gracias a la naturaleza orgánica del precursor de Ti, cuya descomposición durante la calcinación produjo una estructura más porosa por ende un incremento en el área superficial ${ }^{6}$.

Tabla 1. Caracterización fisicoquímica de los catalizadores Ni-Ti-O.

\begin{tabular}{|c|c|c|c|c|c|c|}
\hline \multirow[t]{2}{*}{ Muestra } & \multirow{2}{*}{$\begin{array}{l}\text { Composición } \\
\text { metálica } \\
\text { (mol\%) }\end{array}$} & \multicolumn{2}{|c|}{ Área BET $\left(\mathrm{m}^{2} \mathrm{~g}^{-1}\right)$} & \multirow{2}{*}{$\begin{array}{c}\text { Tamaño } \\
\text { de poro } \\
\text { (nm) }\end{array}$} & \multirow{2}{*}{$\begin{array}{c}\text { Tamaño } \\
\text { de cristal } \\
(\mathrm{nm})\end{array}$} & \multirow{2}{*}{$\begin{array}{l}\text { Parámetro } \\
\text { de red }\left(\mathrm{A}^{\circ}\right)\end{array}$} \\
\hline & & $\begin{array}{c}\text { Antes } \\
\text { de la } \\
\text { reacción }\end{array}$ & $\begin{array}{l}\text { Después } \\
\text { de la } \\
\text { reacción }\end{array}$ & & & \\
\hline $\mathrm{NiO}$ & $\operatorname{Ni}(99.9)$ & 7 & 4 & 10,4 & 30,655 & 4,177 \\
\hline $\mathrm{Ni}_{0.99} \mathrm{Ti}_{0.01} \mathrm{O}$ & $\operatorname{Ni}(99.2): \operatorname{Ti}(0.8)$ & 10 & 10 & 9,5 & 29,159 & 4,177 \\
\hline $\mathrm{Ni}_{0.97} \mathrm{Ti}_{0.03} \mathrm{O}$ & $\operatorname{Ni}(97.4): T i(2.6)$ & 17 & 14 & 11,4 & 25,859 & 4,177 \\
\hline $\mathrm{Ni}_{0.96} \mathrm{Ti}_{0.04} \mathrm{O}$ & $\mathrm{Ni}(96.2): \operatorname{Ti}(3.8)$ & 23 & 23 & 9,5 & 25,723 & 4,181 \\
\hline $\mathrm{Ni}_{0.95} \mathrm{Ti}_{0.05} \mathrm{O}$ & $\mathrm{Ni}(94.7): \operatorname{Ti}(5.3)$ & 46 & 39 & 8,3 & 23,580 & 4,181 \\
\hline $\mathrm{TiO}_{2}$ & $\operatorname{Ti}(99.9)$ & 54 & - & 5,9 & - & - \\
\hline
\end{tabular}

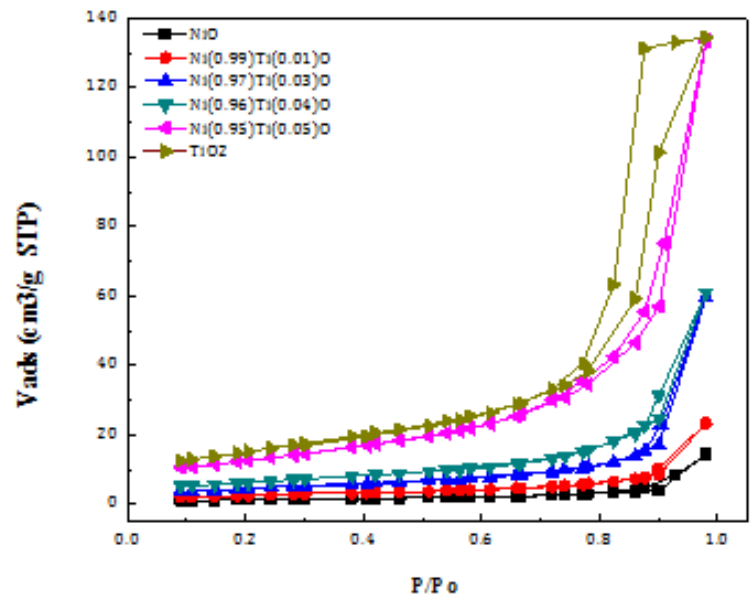

Figura 1. Isotermas de sorción de $\mathrm{N}_{2}$ de los catalizadores Ni-Ti-O.

Los patrones XRD de los catalizadores de Ni-Ti se representan en la figura 2. Se presentaron picos atribuidos a $\mathrm{NiO}$ con estructura de bunsenita que es la fase predominante en todos los catalizadores $\mathrm{Ni}$-Ti con los picos principales: $37,1^{\circ}, 43,3^{\circ}, 62,8^{\circ}, 75,5^{\circ}$ y $79,3^{\circ}$ atribuidos a (111), (200), (220), (311) y (222) planos, respectivamente, de acuerdo con JCPDS 47-1049. Se encontraron también pequeños picos a $35,6^{\circ}$ y $54,0^{\circ}$ correspondientes a la fase rutilo $\mathrm{TiO}_{2}$ (JCPDS 21-1276) y picos a 33,3 $3^{\circ}$ y 49,6 $6^{\circ}$ correspondientes a $\mathrm{NiTiO}_{3}$ (JCPDS 33-0960) en los catalizadores con $\alpha=0,03,0,04$ y 0,05 . $\mathrm{El} \mathrm{TiO}_{2}$ exhibió fuertes picos de difracción situados a $24,8^{\circ}, 37,3^{\circ}, 47,6^{\circ}, 53,5^{\circ}, 55,1^{\circ}$ y $62,2^{\circ}$ correspondientes a la anatasa (JCPDS $21-$ 1272), que es la fase principal formada a través de la calcinación directa del precursor de titanio a $550^{\circ} \mathrm{C}$. Los picos de rutilo también se pueden ver a través de los pequeños picos 
alrededor de $27,4^{\circ}$ y $41,4^{\circ}$ que corresponden a los planos (110) y (111), respectivamente (JCPDS 21-1276). El tamaño de partícula se calculó a partir del pico de difracción mayor (200) usando la fórmula de Scherrer y los resultados se dan en la tabla 1. Se evidenció una correlación inversa entre el área superficial y el tamaño de partícula de los catalizadores como se esperaba. También se puede observar un ensanchamiento en los picos de difracción a mayor contenido de $\mathrm{Ti}$, lo que sugiere que Ti tiende a afectar a la cristalinidad de los catalizadores basados en $\mathrm{Ni}$, y como consecuencia, se espera que las partículas más pequeñas se formen dando áreas superficiales más altas. También se observó una pequeña disminución del parámetro de red, probablemente debido a un dopaje parcial de las especies de $\mathrm{Ti}^{4+}$ en la estructura de $\mathrm{NiO}$.

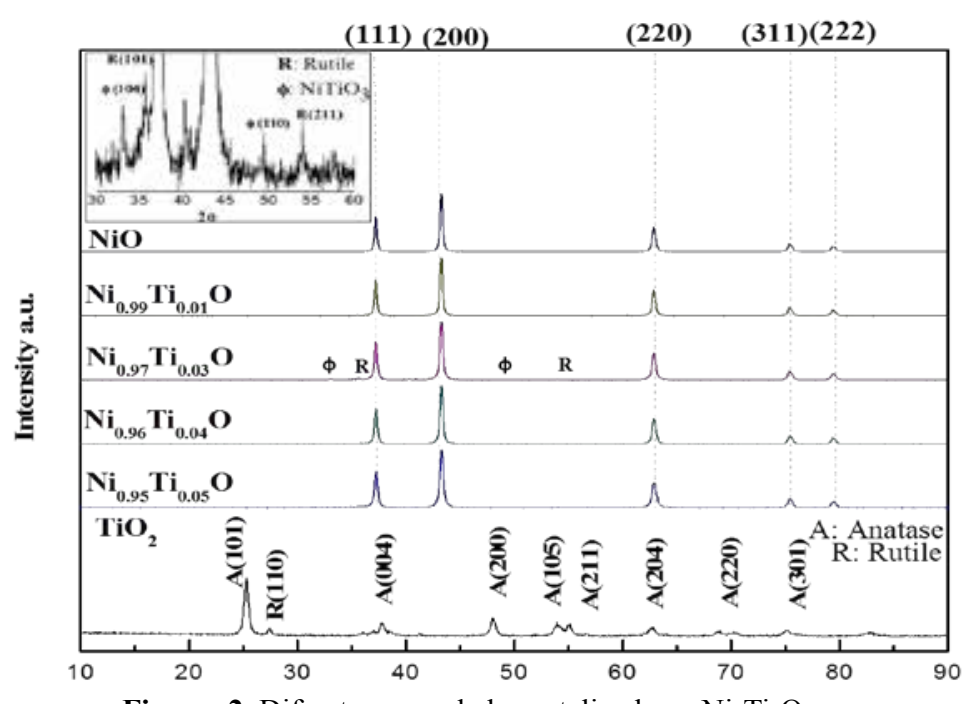

Figura 2. Difractograma de los catalizadores Ni-Ti-O.

\section{2.- Reducción a Temperatura programada (TPR-H $)$}

En la figura 3 se muestra el perfil TPR de los catalizadores de níquel dopados con titanio. El NiO puro muestra un pico de reducción a $421{ }^{\circ} \mathrm{C}$, lo que sugiere la presencia de una sola especie de $\mathrm{Ni}$. El Ni ${ }^{2+}$ es reducido en una sola etapa a níquel metálico de acuerdo a la siguiente ecuación: $\mathrm{NiO}+\mathrm{H}_{2} \rightarrow \mathrm{Ni}+\mathrm{H}_{2} \mathrm{O}$. El test realizado a $\mathrm{TiO}_{2}$ bajo las mismas condiciones del análisis TPR mostró que este compuesto no es reducible en el rango de temperatura trabajado. Sin embargo, la adición de pequeñas cantidades de Ti a la estructura del Ni alteró seriamente el perfil TPR $-\mathrm{H}_{2}$ de los catalizadores. Los óxidos de NiO dopados con Ti mostraron curvas TPR más anchas que se van separando a mayor contenido de Ti y partir $\mathrm{Ni}_{0.97} \mathrm{Ti}_{0.03} \mathrm{O}$ se evidenció la presencia de tres picos de reducción, lo que sugiere la contribución de tres especies reducibles de $\mathrm{Ni}$ en varias formas. Asimismo, los picos se desplazaron a temperaturas más altas, lo que muestra que el Ti tiende a inhibir el proceso de reducción del $\mathrm{Ni}^{2+}$. El hombro ubicado a temperaturas más bajas entre $260-380{ }^{\circ} \mathrm{C}$ es atribuido en la literatura a la reducción de trazas de $\mathrm{Ni}^{3+}$ en el compuesto $\mathrm{Ni}_{2} \mathrm{O}_{3}{ }^{10}$. El pico principal central es atribuido a la reducción 
del $\mathrm{Ni}^{2}+\rightarrow \mathrm{Ni} 0$, mientras que el pico a temperaturas más altas entre 500 a $530^{\circ} \mathrm{C}$ se podría deber a la fuerte interacción de los $\mathrm{Ni}^{2+}$ con $\mathrm{Ti}^{4+}$, dado que la inserción de estas especies en la celda del $\mathrm{Ni}^{\mathrm{O}}$ balancearían las cargas del sistema, haciéndolo más estable y por ende más difícil de reducir. El consumo de hidrógeno disminuyó en proporción al mayor contenido de $\mathrm{Ti}$, lo que es lógico pues hay menos especies de $\mathrm{Ni}^{2+}$ reducibles, a excepción de la muestra con $\mathrm{Ni}_{0.99} \mathrm{Ti}_{0.01} \mathrm{O}$ que no sigue la tendencia y exhibe una drástica disminución del consumo de hidrógeno (tabla 2).

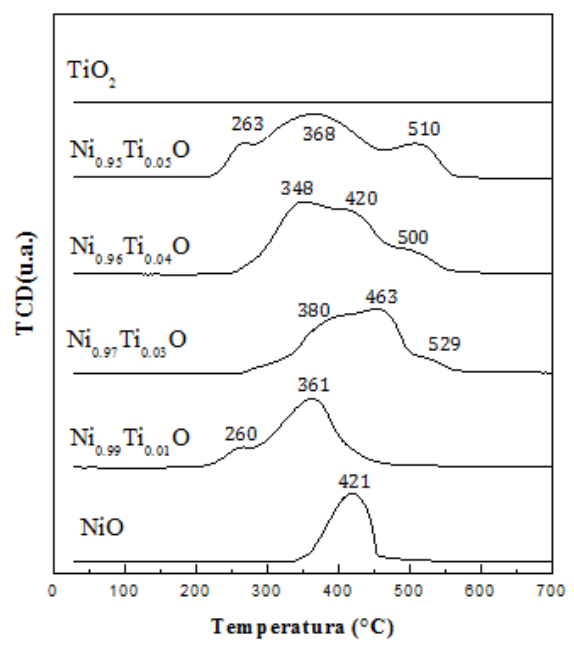

Figura 3. Perfil TPR de los catalizadores Ni-Ti-O.

Tabla 1. Caracterización fisicoquímica de los catalizadores Ni-Ti-O.

\begin{tabular}{|c|c|c|c|c|c|}
\hline \multirow[t]{2}{*}{ Muestra } & \multicolumn{2}{|c|}{$\begin{array}{c}\text { Consumo de } \mathrm{H}_{2} \\
(\mathrm{mmol} / \mathrm{g})\end{array}$} & \multicolumn{3}{|c|}{$\begin{array}{c}\text { Desorción de NH3 } \\
(\mathrm{mmol} / \mathrm{g})\end{array}$} \\
\hline & Teórico & Experimental & MT & AT & $\begin{array}{c}\text { Acidez } \\
\text { Total }\end{array}$ \\
\hline $\mathrm{NiO}$ & 13,39 & 13,28 & 38,33 & 17,36 & 55,69 \\
\hline $\mathrm{Ni}_{0.99} \mathrm{Ti}_{0.01} \mathrm{O}$ & 13,38 & 10,57 & 29,86 & 21,79 & 51,65 \\
\hline $\mathrm{Ni}_{0.97} \mathrm{Ti}_{0.03} \mathrm{O}$ & 13,36 & 13,27 & 64,34 & 32,23 & 96,57 \\
\hline $\mathrm{Ni}_{0.96} \mathrm{Ti}_{0.04} \mathrm{O}$ & 13,35 & 12,98 & 50,41 & 36,58 & 86,99 \\
\hline $\mathrm{Ni}_{0.95} \mathrm{Ti}_{0.05} \mathrm{O}$ & 13,34 & 11,69 & 41,34 & 42,92 & 84,26 \\
\hline $\mathrm{TiO}_{2}$ & - & . & 26,66 & 20,32 & 47,01 \\
\hline
\end{tabular}

\section{3.- Análisis Termogravimétrico (TGA)}

Como se observa en la figura 4, la pérdida de peso para los precursores de $\mathrm{NiO}$ y $\mathrm{Ni}_{0.95} \mathrm{Ti}_{0.05} \mathrm{O}$ ocurrió en el mismo número de etapas. Para la formación de $\mathrm{NiO}$ a partir de $\mathrm{Ni}\left(\mathrm{NO}_{3}\right) \cdot 6 \mathrm{H}_{2} \mathrm{O}$, se identificaron cuatro etapas principales y la pérdida de peso en cada etapa corresponde a los productos liberados por las reacciones de descomposición térmica de $\mathrm{Ni}\left(\mathrm{NO}_{3}\right)_{2} \cdot 6 \mathrm{H}_{2} \mathrm{O}$ propuestas por Brockner ${ }^{11}$. La primera etapa $(9,82 \%$ en peso) que se produce en el intervalo de temperatura de $60^{\circ}-100^{\circ} \mathrm{C}$ corresponde a la exclusión de un equivalente de agua y algunas 
trazas de disolvente. La segunda pérdida de peso $\left(18,15 \%\right.$ en peso) entre $100^{\circ}-224^{\circ} \mathrm{C}$ se asignó a la eliminación de tres equivalentes de $\mathrm{H}_{2} \mathrm{O}$. La tercera etapa en el intervalo de $224^{\circ}-332{ }^{\circ} \mathrm{C}$ con una pérdida de peso de $38,33 \%$ se asignó a la eliminación de $\mathrm{NO}_{2}, \mathrm{HNO}_{3} \mathrm{y}$ $\mathrm{H}_{2} \mathrm{O}$ a través de la descomposición de $\mathrm{Ni}\left(\mathrm{NO}_{3}\right)_{2} \cdot 2 \mathrm{H}_{2} \mathrm{O}$. La cuarta etapa $(2,67 \%$ en peso) se puede atribuir a la descomposición de óxido de $\mathrm{Ni}_{2} \mathrm{O}_{3}$ a $\mathrm{NiO}$. Para la síntesis de $\mathrm{Ni}_{0.95} \mathrm{Ti}_{0.05} \mathrm{O}$ se observó el mismo número de pasos. La primera pérdida de masa $(7,04 \%$ en peso) se debe a la eliminación del agua del precursor de $\mathrm{Ni}$ y a la hidrólisis del precursor de Ti. La segunda etapa mostró una mayor pérdida de masa $(33,73 \%)$ en comparación con la curva $\mathrm{Ni}\left(\mathrm{NO}_{3}\right)_{2} \cdot 6 \mathrm{H}_{2} \mathrm{O}$ que podría estar asociada a la liberación de $\mathrm{CH}_{3} \mathrm{OH}$ a partir de la hidrólisis de $\mathrm{Ti}\left[\mathrm{N}\left(\mathrm{CH}_{2} \mathrm{CH}_{2} \mathrm{O}\right)_{3}\left(\mathrm{CH}_{3} \mathrm{O}\right)\right]^{20}$ y la exclusión de agua y $\mathrm{NO}_{2}$ de la descomposición parcial de $\mathrm{Ni}\left(\mathrm{NO}_{3}\right)_{2} .2 \mathrm{H}_{2} \mathrm{O}$. La tercera pérdida de masa $(29,54 \%)$ corresponde a la descomposición total de la fase $\mathrm{Ni}\left(\mathrm{NO}_{3}\right)(\mathrm{OH})_{1.5} \mathrm{O}_{0.25} . \mathrm{H}_{2} \mathrm{O}$ a Ni $\mathrm{O}_{3}{ }^{19}$ y la eliminación del ligando orgánico del precursor de Ti. La última etapa $(2,22 \%)$ en el intervalo de $310^{\circ}$ a $450^{\circ} \mathrm{C}$ podría atribuirse a la descomposición de óxido de $\mathrm{Ni}_{2} \mathrm{O}_{3}$ a fase $\mathrm{NiO}$ considerando que el óxido de Ti se descompuso en la etapa anterior. Por otra parte, la gráfica termogravimétrica mostró una curva casi plana más allá de $450^{\circ} \mathrm{C}$ para ambos catalizadores, lo que indica que la temperatura de calcinación utilizada fue apropiada para la síntesis del catalizador $\left(550^{\circ} \mathrm{C}\right)$ para asegurar la calcinación total de la fase orgánica y la formación de la fase cristalina.

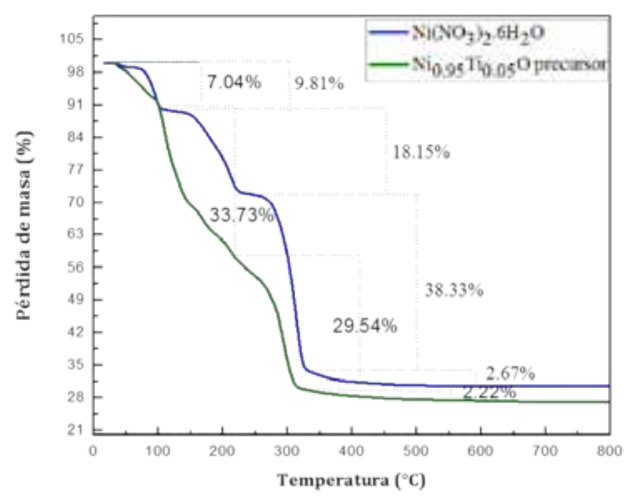

Figura 4. Termograma de los precursores de $\mathrm{NiO}$ y $\mathrm{Ni}_{0.95} \mathrm{Ti}_{0.05}$.

\section{4.- Espectrosocopía Raman}

Los espectros Raman de los catalizadores de níquel dopados con Ti se representan en la figura 5. El óxido de níquel exhibió un pico principal a aproximadamente $504 \mathrm{~cm}^{-1}$ atribuido al modo de fonón óptico (TO) de primer orden y se origina a partir de los defectos en el cristal de $\mathrm{NiO}$ y otro menos intenso a $720 \mathrm{~cm}^{-1}$ atribuido al fonón longitudinal óptico. Los picos a $198 \mathrm{~cm}^{-1}(\mathrm{Eg}), 396 \mathrm{~cm}^{-1}\left(\mathrm{~B}_{1 \mathrm{~g}}\right), 515 \mathrm{~cm}^{-1}\left(\mathrm{~A}_{1 \mathrm{~g}}\right)$ y $638 \mathrm{~cm}^{-1}\left(\mathrm{E}_{\mathrm{g}}\right)$ se asignaron a la fase anatasa con el grupo espacial $\mathrm{D}_{4 \mathrm{~h}}$ para el $\mathrm{TiO}_{2}$. No se encontraron picos de fase rutilo probablemente debido a que los picos más intensos de la anatasa ocultaron los picos rutilo. La presencia de pequeñas cantidades de Ti cambió dramáticamente los espectros de NiO. Los picos a 319, 
446 y $587 \mathrm{~cm}^{-1}$ correspondientes a la fase rutilo de $\mathrm{TiO}_{2}$ aparecieron en los espectros 112 , mientras que los picos de $\mathrm{NiO}$ a $504 \mathrm{~cm}^{-1}$ y $721 \mathrm{~cm}^{-1}$ cambiaron a $511 \mathrm{~cm}^{-1}$ y $750 \mathrm{~cm}^{-1}$, respectivamente, lo que sugiere la fuerte interacción entre Ti y NiO. Estos resultados están de acuerdo con los resultados XRD y TPR.

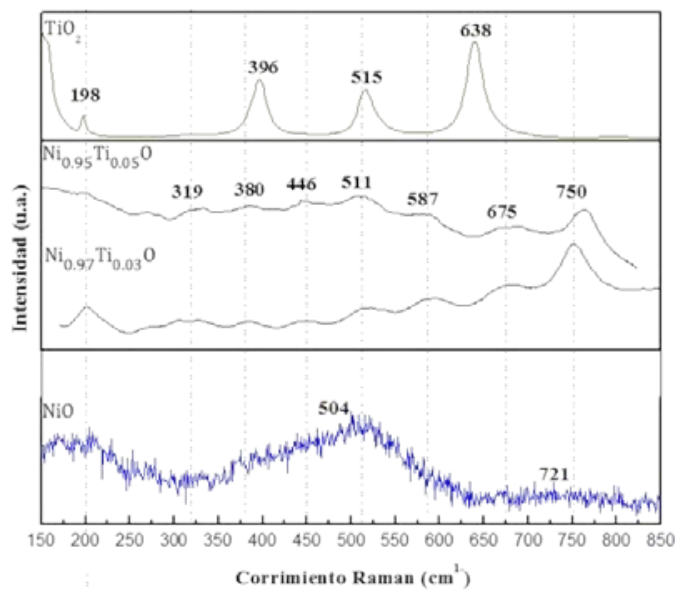

Figura 5. Espectros Raman de los catalizadores Ni-Ti-O.

\section{5.- Desorción a Temperatura Programada de $\mathrm{NH}_{3}\left(\mathrm{TPD}-\mathrm{NH}_{3}\right)$}

La cooperación de sitios ácidos con sitios básicos es sumamente importante en reacciones particular e influye mucho en la selectividad de las reacciones, por tanto es necesario saber cómo las fuerzas de los sitios ácidos están distribuidas. Para caracterizar la naturaleza de los sitios ácidos se usó la desorción a temperatura programada de $\mathrm{NH}_{3}$. Los picos de desorción en rangos de temperatura máxima de 180 a $250^{\circ} \mathrm{C}(\mathrm{BT}), 280-330^{\circ} \mathrm{C}(\mathrm{MT})$ y $380-500^{\circ} \mathrm{C}(\mathrm{AT})$ normalmente se atribuyen a $\mathrm{NH}_{3}$ quimisorbido en sitios ácidos débiles, medianos y fuertes, respectivamente. Las muestras presentan un perfil con dos picos de desorción (figura 6). El pico de menor temperatura correspondiente a la acidez intermedia ubicada entre 320$330{ }^{\circ} \mathrm{C}$ y el pico de mayor temperatura de acidez fuerte ubicado en el rango entre $450-490$ ${ }^{\circ} \mathrm{C}$, aproximadamente. Se observó que la adición de pequeñas cantidades de Ti provocó el aumento de sitios ácidos en comparación al $\mathrm{NiO}$ puro. Asimismo, se observó un aumento progresivo de la acidez fuerte a mayor contenido de $\mathrm{Ti}^{4+}$. 


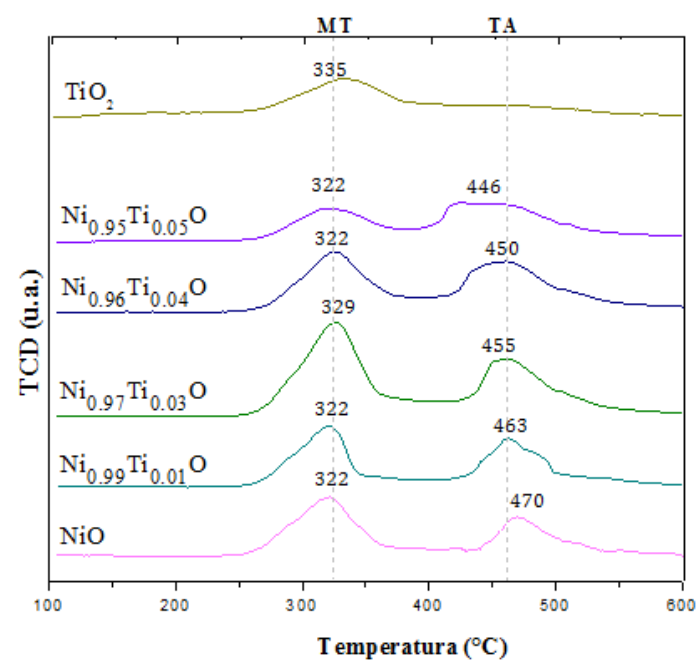

Figura 6. Perfil TPD-NH $\mathrm{NH}_{3}$ de los catalizadores Ni-Ti-O.

\section{6.- Desorción a Temperatura Programada de $\mathrm{O}_{2}\left(\mathrm{TPD}_{-} \mathrm{O}_{2}\right)$}

Las mediciones de TPD-O $\mathrm{O}_{2}$ con adsorción previa de $\mathrm{O}_{2}$ (figura 7) se realizaron para evaluar el tipo de oxígeno superficial y sus propiedades que podrían dar algunas luces para entender el proceso de oxidación catalítica. Se registró la liberación de oxígeno del catalizador de $25^{\circ} \mathrm{C}$ a $850^{\circ} \mathrm{C}$. El perfil de desorción de oxígeno para los catalizadores $\mathrm{Ni}$-Ti-O, exhibió tres picos correspondientes a tres especies de oxígeno. Según Iwamoto et al. ${ }^{12}$, el oxígeno molecular se adsorbe sobre la superficie de óxidos metálicos formando superóxidos e iones peróxido. Los cuatro estados diferentes de oxígeno adsorbidos a intervalos de temperaturas crecientes son: $\alpha\left(30-40{ }^{\circ} \mathrm{C}\right), \beta\left(320-360{ }^{\circ} \mathrm{C}\right), \gamma\left(420-450{ }^{\circ} \mathrm{C}\right)$ y $\delta$ (por encima de $520^{\circ} \mathrm{C}$ ) correspondientes $\mathrm{a} \mathrm{O}_{2}, \mathrm{O} 2-, \mathrm{O}-\left(\right.$ para $\gamma \mathrm{y} \delta$ ), respectivamente ${ }^{12}$. Por lo tanto, según la literatura los tres picos compilados por los catalizadores corresponden a las especies $\beta, \gamma$ y $\delta$. El $\mathrm{NiO}$ exhibió un gran pico de desorción de oxígeno a una temperatura máxima de $700{ }^{\circ} \mathrm{C}$, correspondiente a un estado de oxígeno no estequiométrico O-. Cuando O- y $\mathrm{O}_{2}$ - están presentes en la superficie del catalizador, facilitan la oxidación total del hidrocarburo debido a la naturaleza electrofílica de estos iones de oxígeno que atacan directamente a la molécula orgánica en la región de su más alta densidad electrónica (enlaces $\Pi$ ). Por otro lado, es probable que especies como $\mathrm{O}_{2}$ - conduzcan a la producción de productos oxigenados a través de un ataque nucleofílico. Debido a sus propiedades no oxidantes, la presencia de iones $\mathrm{O}_{2}$ - promueve una reacción más selectiva $^{13 .}$

La cantidad de oxígeno desorbido mostrada en la tabla 2 indica una caída apreciable de la liberación de oxígeno con un mayor contenido de Ti, comenzando con 244,89 mmol/g de $\mathrm{O}_{2}$ para $\mathrm{NiO}$ hasta $108,52 \mathrm{mmol} / \mathrm{g}$ de $\mathrm{O}_{2}$ para $\mathrm{Ni}_{0.95} \mathrm{Ti}_{0.05} \mathrm{O}$. Además, el pico asignado a especies $\delta$ disminuyó en intensidad y se desplazó a temperaturas más bajas hasta una señal débil, que se registró para la muestra $\mathrm{Ni}_{0.95} \mathrm{Ti}_{0.05}$. Este resultado muestra que las especies de Ti disminuyen 
notablemente la presencia de oxígeno no estequiométrico causado por las vacantes catiónicas en $\mathrm{NiO}$. De acuerdo con Heracleous et al. ${ }^{6}$, la inserción de iones con mayor valencia que $\mathrm{Ni}^{2+}$ como $\mathrm{Ti}^{4+}$ disuelven los huecos positivos $\mathrm{p}^{+}$a través del llenado de las vacantes catiónicas de NiO. Por lo tanto, se obtiene una superficie con menos defectos que absorbe menos cantidad de oxígeno ${ }^{14}$. Como las especies electrofílicas $\mathrm{O}_{2}$ - y O- se encuentran en menor cantidad, hay menor capacidad de causar una oxidación profunda de la olefina, por lo que se espera que alcancen mayores niveles de selectividad.

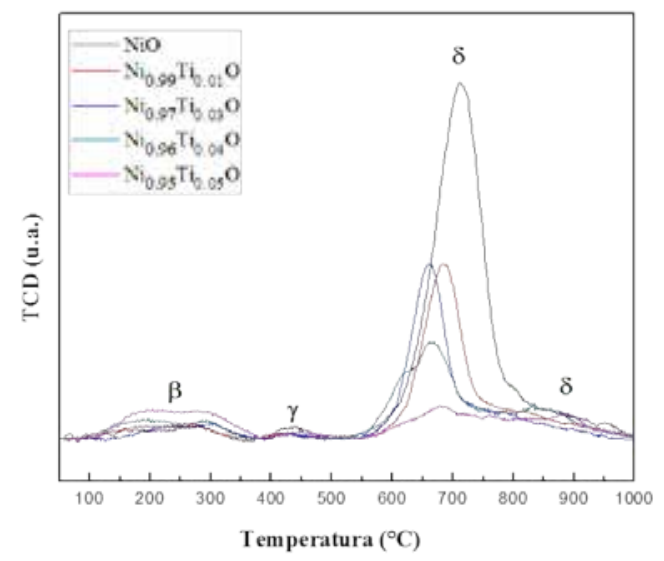

Figura 7. Perfil TPD-O2 de los catalizadores Ni-Ti-O.

\section{Ensayos catalíticos}

Los ensayos catalíticos se realizaron dentro de un intervalo de temperatura de $300-450{ }^{\circ} \mathrm{C}$ con una constante de $\mathrm{W} / \mathrm{F}=0,48$ g.s $/ \mathrm{mL}$ y los resultados se muestran en la figura 8. Los resultados de conversión de etano con el aumento de temperatura mostraron que la adición de $\mathrm{Ti}$ en la estructura del $\mathrm{NiO}$ tuvo un efecto positivo en términos de actividad. A pesar que áreas superficiales relativamente bajas se obtuvieron en general, se alcanzaron altos niveles de conversión de etano que aumentó con una mayor composición de Ti en la muestra. Estos resultados podrían estar asociados al incremento en el área superficial reportado anteriormente, así como la presencia de sitios ácidos nuevos causados por el desequilibrio de la carga en los enlaces Ni-Ti-O que favorece la adsorción de la parafina. La capacidad reductora de los catalizadores también tuvo un papel importante: la reducibilidad del $\mathrm{NiO}$ se vio afectada con la adición de Ti causando una distribución de las especies de Ni que se desplazaron a temperaturas bajas y altas. Las especies desplazadas a temperaturas más bajas podrían ser responsables a la mejor reducción de la superficie del catalizador. Los experimentos de selectividad se realizaron a temperatura constante de $400{ }^{\circ} \mathrm{C}$ variando $\mathrm{W} / \mathrm{F}$ de 0,24 a 2,4 g.s/mL (figura 5). Los resultados indicaron que el Ti también tuvo un efecto positivo en la selectividad de los catalizadores. La selectividad mejoró de $35 \%$ de $\mathrm{NiO}$ a aproximadamente $77 \%$ para $\mathrm{Ni}_{0.95} \mathrm{Ti}_{0.05} \mathrm{O}$, todos los catalizadores experimentaron una declinación en los niveles de selectividad de menos de 15\% dentro de la gama de conversión. Es importante destacar que los catalizadores fueron capaces de lograr buenos niveles de 
selectividad a los niveles de conversión moderados. El aumento de los niveles de selectividad podría atribuirse a la reducción dramática del oxígeno no estequiométrico en la red del $\mathrm{NiO}$ ya que la presencia de especies electrofílicas de oxígeno $\left(\mathrm{O}-\mathrm{y} \mathrm{O}_{2}-\right)$ en la superficie de la $\mathrm{NiO}$ son las responsables de la oxidación total de etano. El dopante promotor $\mathrm{Ti}^{4+}$, debido a su valencia más elevada, podría ser beneficial para reducir los efectos positivos creados en el óxido no estequiométrico de $\mathrm{NiO}$. Este fenómeno ha sido abordado antes por varios grupos ${ }^{15}$. La mejora de selectividad también es pertinente a los resultados de TPR que revelaron la presencia de alta temperatura especies de Ni reducibles que la disminución de la labilidad del oxígeno en la superficie, pero de una manera controlada, porque una gran cantidad de enlaces fuertes Ni-O en la superficie tendría un efecto negativo en la actividad de los catalizadores que no se exhiben en los experimentos. La medida de las áreas BET de los catalizadores de Ni-Ti-O, después de la reacción, no mostraron una reducción significativa de la superficie, lo que sugiere que no se dio la formación de coque (o en todo caso, fue insignificante) durante la reacción, sin embargo, se requieren estudios de estabilidad térmica (tabla 1).

De los resultados obtenidos en los experimentos, se pudo observar que el Ti tuvo un efecto promotor en la primera etapa del mecanismo, es decir Mars-Van Krevelen, pues tiene la propiedad de debilitar suficientemente el enlace Ni-O para facilitar la reducción de la superficie, pero al mismo tiempo hace el enlace $\mathrm{Ni}-\mathrm{O}$ lo suficientemente fuerte para evitar la profunda oxidación del etileno. La temperatura de calcinación también afectó al rendimiento de los catalizadores; en lugar de ceder catalizadores con áreas de superficie alta que produjo catalizadores con sitios ácidos fuertes que mejoró la conversión de etano, pero el área pequeña podría haber limitado el acceso de la olefina para promover las reacciones secundarias.
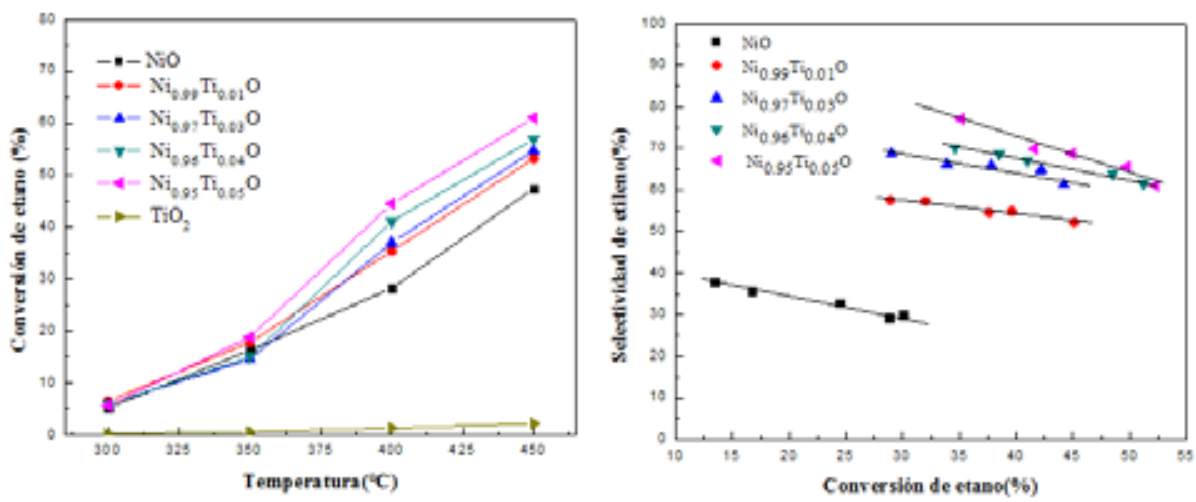

Figura 8. Ensayos catalíticos de los catalizadores: a) conversión de etano vs. Temperatura con $\mathrm{W} / \mathrm{F}=0,48 \mathrm{gs} / \mathrm{mL}$ y b) selectividad de etileno Vs. Conversión de etano a $400{ }^{\circ} \mathrm{C}$. 


\section{CONCLUSIONES}

Los catalizadores sintetizados a base de $\mathrm{NiO}$ dopados con Ti mostraron un efecto muy positivo en la mejora del rendimiento de los catalizadores para conseguir buenos niveles tanto de actividad como de selectividad. El Ti y Ni resultaron trabajar sinérgicamente para promover la generación de sitios ácidos y una redistribución de especies reducibles a bajas y altas temperaturas que conducen a la conversión de etano y un agotamiento de los oxígenos no estequiométricos, obteniendo un catalizador más selectivo hacia el etileno. La actividad de los catalizadores parece no verse afectada por las superficies bajas obtenidas y más bien basarse en la acidez conseguida. Este hecho resalta el efecto de la temperatura de calcinación y el método de síntesis utilizado para la fabricación de los catalizadores Ni-Ti.

\section{AGRADECIMIENTO}

Los autores agradecen el apoyo financiero para este trabajo proporcionado por el Programa INNOVATE PERÚ (Convenio $\mathrm{N}^{\circ}$ 229-FINCyT-2013), a la Universidad Nacional de Ingeniería - Facultad de Ciencias y al Instituto General de Investigación de la Universidad Nacional de Ingeniería (IGI), por el apoyo financiero para nuestro grupo de investigación.

\section{REFERENCIAS BIBLIOGRÁFICAS}

1. GlobalData [Internet]. 2016 [citado el 02 Jun 2016]. Disponible en: http://store. globaldata.com/market-reports/Oil- -Gas

2. Gartner C, Van Vee A, Lercher J. Oxidative Dehidrogenation of Ethane: Common Principles and Mechanistic Aspects. ChemCatChem. 2013;5(11):3197-3198.

3. Cavani F, Trifiró F. The oxidative dehydrogenation of ethane and propane as an alternative way for the production of light olefins. Catal Today. 1995;24:307-313.

4. Boukhlouf H, Barama A, Benrabaa R, Guerrero Caballero J, Löfberg A, BordesRichard E. Catalytic activity in the oxidative dehydrogenation of ethane over $\mathrm{Ni}$ and/ or Co molybdate catalysts: Synthesis and characterization, Comptes Rendus Chimie. 2017;20(1): 30-39.

5. Zhu D, Rosenfeld D, Anjum S, Sangaru Y, Saih S, Ould-Chilk S, et al. Ni-Ta-O mixed oxide catalysts for the low temperature oxidative dehydrogenation of ethane to ethylene. J Catal. 2015;329:291-306.

6. Heracleous E, Lemonidou A. Ni-Nb-O mixed oxides as highly active and selective catalysts for ethane production via ethane oxidative dehydrogenation. Part I: Characterization and Catalytic performance. J Catal. 2006;231:162-174.

7. Brussino P, Bortolozzi JP, Milt VG, Banús ED, Ulla MA. Ni/Ce $/ \gamma-\mathrm{Al} 2 \mathrm{O} 3$ coated onto cordierite monoliths applied to Oxidative Dehydrogenation of Ethane (ODE). Catal Today. 2016;273:259-265. 
8. Skoufa Z, Xantri G, Heracleous E, Lemonidou AA. A study of Ni-Al-O mixed oxides as catalysts for the oxidative conversion of ethane to ethylene. Appl Catal A. 2014;471:107117.

9. Heracleous E, Lemonidou AA. Ni-Me-O mixed metal oxides for the effective oxidative dehydrogenation of ethane to ethylene-Effect of promoting metal Me. J Catal. 2010;270:67-75.

10. Kotsev N, Ileva L. Determination of non-stoichiometric oxygen in NiO by temperatureprogrammed reduction. Catal Letters. 1993;18:173-176.

11. Brockner W, Ehrhaurdt C, Gjikaj M. Thermal decomposition of nickel nitrate hexahydrate, $\mathrm{Ni}(\mathrm{NO} 3) 2.6 \mathrm{H} 2 \mathrm{O}$, in comparison to $\mathrm{Co}\left(\mathrm{NO}_{3}\right) 2.6 \mathrm{H}_{2} \mathrm{O}$ and $\mathrm{Ca}\left(\mathrm{NO}_{3}\right)_{2} \cdot 4 \mathrm{H}_{2} \mathrm{O}$. Thermochim Acta. 2007;456: 64-68.

12. Iwamoto M, Yoda Y, Egashira M, Seiyama T. Study of Metal Oxide Catalysis by Temperature Programmed Desorption. Chemisorption of Oxygen on Nickel Oxide. J Phys Chem. 1976;80(18): 1989.

13. Guang-Rui G, Ying-Ai L, Yan-Chun T, Zhi H, Jun-Jie L, Hong Y, et al. Investigation on the structure of TiO2 films sputtered on alloy substrates.Vacuum. 2003;71:487-490.

14. Heracleous E, Lemonidou AA. Ni-Me-O mixed metal oxides for the effective oxidative dehydrogenation of ethane to ethylene-Effect of promoting metal Me. J Catal. 2010;270:67-75.

15. Zhu H, Dong H, Laveille P, Saih Y, Caps V, Basset JM. Metal oxides modified NiO catalysts for oxidative dehydrogenation of ethane to ethylene. Catal Today. 2014;228:5864. 